\title{
Power Reduction of Josephson Random Access Memory Using Stochastic Resonance
}

\author{
Ryosuke Kihara, Yuki Yamanashi, Member, IEEE, and Nobuyuki Yoshikawa, Member, IEEE
}

\begin{abstract}
A superconducting quantum interference device (SQUID) is used as a memory cell in a superconducting Josephson random access memory (RAM) system. In the Josephson RAM, power is mainly consumed by driver circuits, which drive word and bit lines to select the target memory cell. We investigated power reduction of read/write operation of the SQUID memory cell by using a stochastic resonance phenomenon. By using a noise-assisted state transition of the SQUID, the driving current required for read/write operation of the memory cell can be reduced. We investigated optimum bias and noise conditions where stochastic resonance efficiently occurs on the basis of analog circuit simulation that takes influences of thermal noises into account. According to the simulation results, the power dissipation required for read/write operation can be reduced by approximately $20 \%$ under the optimum bias and noise conditions when the error rate of read/write operation is $10^{-10}$. We designed a 1-bit rf-SQUID memory cell using the AIST $2.5 \mathrm{kA} / \mathrm{cm}^{2} \mathrm{Nb}$ standard process 2 , and tested the cell by applying a white noise from the room-temperature instrument. The probability that a datum is written to the memory cell and read-out correctly was measured under various bias and noise conditions. We have experimentally observed stochastic resonance and obtained the error rate of $10^{-5}$ when the power dissipation of read/write operation is reduced to $80 \%$ compared to the conventional Josephson RAM.
\end{abstract}

Index Terms - Josephson RAM, memory, stochastic resonance, rf-SQUID, SFQ circuit

\section{INTRODUCTION}

$\mathrm{T}$ HE performance of the computation system has been improved for several decades. At the present time, the performance of the supercomputer reaches 93.0 PFLOPS [1]. However, power consumption of such high-performance computer is more than $10 \mathrm{MW}$. Power consumption of the next-generation supercomputer that has the performance of 1 Exa-FLOPS system is estimated to reach several hundred MW. It is not practical to build such a large power system.

Energy efficiency of the superconducting circuits is more than three orders of magnitude higher than that of the

Manuscript received XXXX XX, XXXX; accepted XXXX XX, XXXX. Date of publication XXXX XX, XXXX; date of current version XXXX XX, XXXX. This work was supported in part by JSPS KAKENHI Grant Number 25820137 and 26220904.

Ryosuke Kihara, Yuki Yamanashi, and Nobuyuki Yoshikawa are with the Department of Electrical and Computer Engineering, Yokohama National

University, Yokohama 240-8501, Japan (e-mail: yamanasi@ynu.ac.jp).

Color versions of one or more of the figures in this paper are available online at http://ieeexplore.ieee.org.

Digital Object Identifier XXXXXXXXXXXXXXXXX). semiconductor integrated circuit [2, 3]. High energy efficiency of the superconducting circuits have been demonstrated [4-8]. In the superconducting computation system, memory access is thought to be the main origin of power consumption because of driving of word and bit lines for the random access memory (RAM) [9-12]. To reduce total power consumption of the computation systems based on the superconducting circuit, reduction in power consumption of memory access is efficient. Recently, RAM cells based on novel materials have been proposed and implemented for superconducting computation systems [13-16]. Power reduction of memory access benefits not only conventional Josephson RAM but also these novel RAMs.

In this study, we investigated power reduction in the superconducting RAM by introducing stochastic resonance [17]. By using stochastic resonance, signal to noise ratio of the sensing circuit can be improved [17]. It is known that stochastic resonance occurs in a superconducting circuit [18]. We investigated use of stochastic resonance to reduce the driving current for datum read/write operation for the superconducting RAM. We derived the appropriate noise condition to efficiently obtain stochastic resonance in the memory cell of the superconducting RAM. We implemented and tested the memory cell by applying white noise from a room-temperature instrument.

\section{State Transition of SQuid by Stochastic Resonance}

Fig. 1 shows an equivalent circuit of an rf-SQUID comprising of one Josephson junction. The rf-SQUID is bistable device and thus can be used as a memory cell for the superconducting RAM [9, 10]. Potential energy of the rf-SQUID is represented by

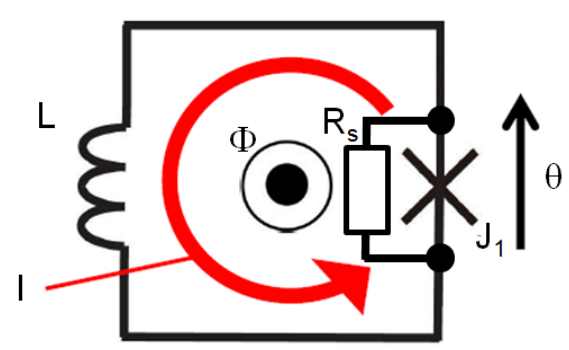

Fig.1. Equivalent circuit of an rf-SQUID. L is inductance consisting rf-SQUID loop. $\mathrm{J}_{1}$ is Josephson junction. $\Phi$ is internal magnetic flux in rf-SQUID, $I$ is the circular current. $\mathrm{R}_{\mathrm{S}}$ is shunt resistance of Josephson junction $\mathrm{J}_{1} . \theta$ is the phase difference of the macroscopic wave function across the Josephson junction $\mathrm{J}_{1}$. 


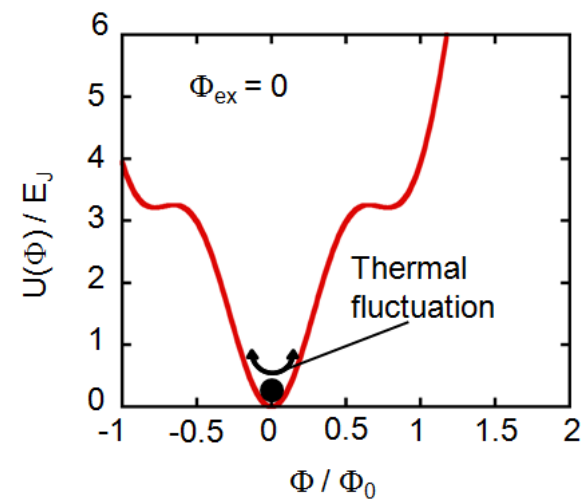

(a)

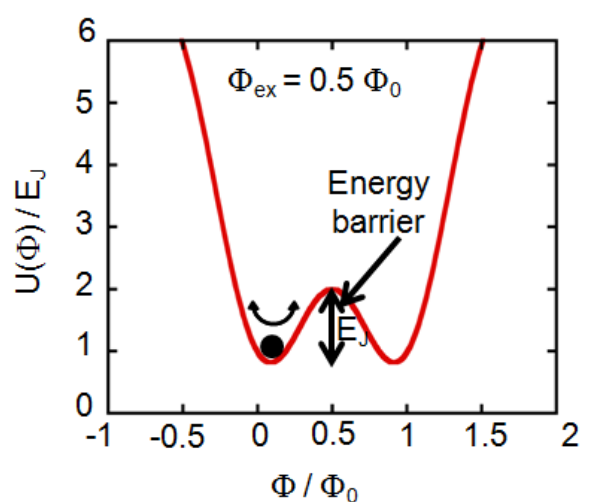

(b)

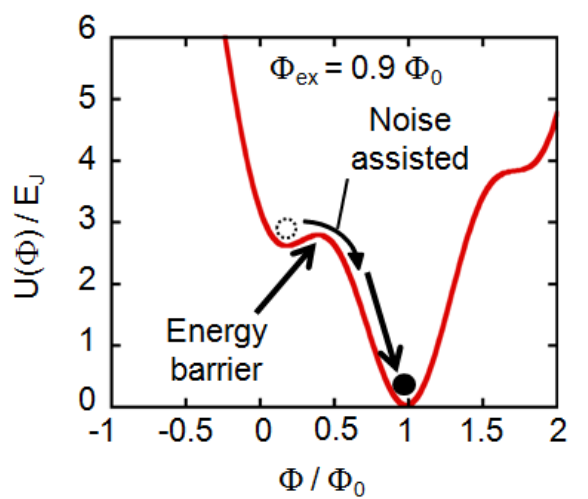

(c)

Fig. 2. Potential energy diagrams of rf-SQUID. (a) $\Phi_{\mathrm{ex}}=0$, (b) $\Phi_{\mathrm{ex}}=0.5 \Phi_{0}$, (c) $\Phi_{\mathrm{ex}}=0.9 \Phi_{0} . \Phi_{\mathrm{ex}}$ is the external magnetic flux uniformly applying to whole of the rf-SQUID, $\Phi_{0}=2.07 \times 10^{-15} \mathrm{~Wb}$ is single flux quantum, $\mathrm{E}_{\mathrm{J}}$ is Josephson energy. $L=30.1 \mathrm{pH}, J_{l}=50 \mu \mathrm{A}$ in Fig. 1 . $\mathrm{E}_{\mathrm{J}}=1.68 \times 10^{-20} \mathrm{~J}$, corresponds to 1200 $\mathrm{K}$ thermal energy.

$$
\begin{aligned}
& U(\Phi)=(1 / 2) L I^{2}+E_{J}(1-\cos \theta) \\
& \left.\quad=\left\{\left(\Phi-\Phi_{e x}\right) / 2 L\right\}+E_{J}\left[1-\cos \left\{2 \pi\left(\Phi-\Phi_{e x}\right) / \Phi_{0}\right)\right\}\right]
\end{aligned}
$$

where $L$ is inductance of the rf-SQUID loop, $I$ is the superconducting current flowing in the rf-SQUID, $\Phi$ is the internal magnetic flux, $\Phi_{\mathrm{ex}}$ is the external magnetic flux applied to the rf-SQUID, $E_{J}$ is Josephson energy when no current is flowing in the junction, $\theta$ is phase difference of the macroscopic wave function across the Josephson junction, and $\Phi_{0}$ is a magnetic flux quantum in a superconductor.

Fig. 2 shows noise-assisted state transition of the rf-SQUID, which corresponds to datum write operation for the SQUID memory cell in this study. The circuit parameters were optimized to obtain the stochastic resonance characteristic. In the initial state, where no magnetic flux is applied to the SQUID, energy potential of the SQUID has one minimum and thus the state is stable (Fig. 2(a)). When the external magnetic flux is applied, two potential minima appear according to eq. (1) as shown in Fig. 2(b). These two potential minima correspond to ' 0 ' and ' 1 ' sates in the memory cell, respectively. Because these minima are isolated by a potential barrier, state transition does not occur. Fig. 2(c) shows potential energy of the SQUID when the magnetic flux of $0.9 \Phi_{0}$ is applied. In this case, potential barrier that isolates two stable states is low and the state can transit from the ' 0 ' state to the ' 1 ' state if appropriate noises exist. In this noise-assisted transition, the required magnetic flux is less than $\Phi_{0}$, whereas magnetic flux of more than $\Phi_{0}$ is required to induce state transition from ' 0 ' to ' 1 ' states in the conventional SQUID memory.

We simulated read/write operation of the rf-SQUID memory cell taking thermal noises into account using the circuit simulator, JSIM_N [19]. In simulation, datum ' 1 ' is written to the SQUID by inputting square wave shaped magnetic flux with the amplitude of $\Phi_{a m p}$ and the datum is read-out 10000 times at the frequency of $1 \mathrm{GHz}$ assuming existence of a thermal noise that has the bandwidth of $10 \mathrm{THz}$. We defined the probability that the datum is written to the memory cell and the datum is read-out correctly as a success rate. We calculated the success rate of the SQUID memory read/write operation under various noise and magnetic flux conditions by changing temperature $T$ and $\Phi_{\text {amp }}$. Fig. 3 shows calculated dependences of success rates on noise energy. When the noise is too small, the success rate is low because the noise assisted transition does not occur. The highest success rate is obtained under the optimum noise condition. On the other hand, the success rate is deteriorate with increase in noise energy because state returning from ' 1 ' to the ' 0 ' and the input of two flux quanta occurs by large noise energy. This is the typical characteristic of stochastic resonance [17].

The simulation result shows the success rate of 1.000 is obtained under the appropriate noise condition where the ratio of noise energy and Josephson energy $\left(\mathrm{k}_{\mathrm{B}} \mathrm{T} / \mathrm{E}_{\mathrm{J}}\right)$ is $0.16-1.08$. when $\Phi_{a m p}$ is $0.45 \Phi_{0}$, power consumption for read/write operation of the SQUID memory cell can be reduced by approximately $20 \%$ because required $\Phi_{a m p}$ for write/read

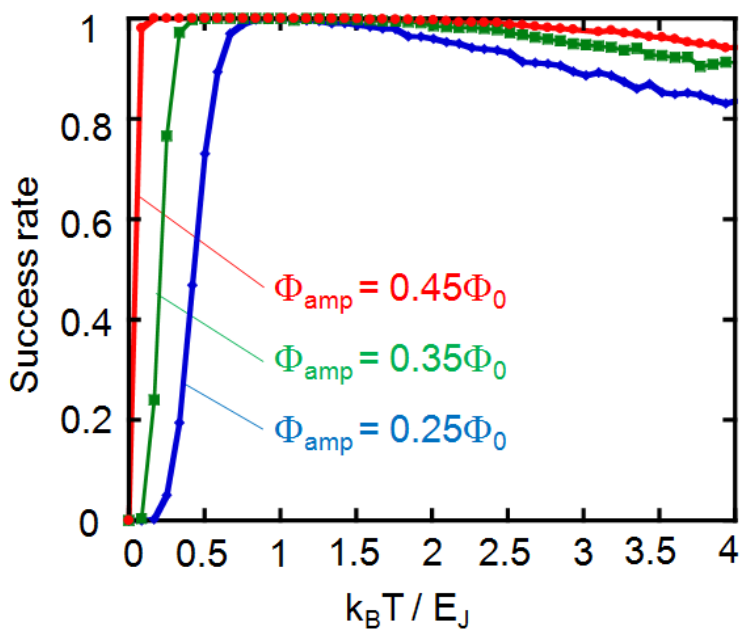

Fig. 3. Simulated dependences of the success rate of the read/write operation of the rf-SQUID memory cell on the noise energy when $\Phi_{\text {amp }}$ is $0.25 \Phi_{0}$, $0.35 \Phi_{0}$ and $0.45 \Phi_{0}$. The horizontal axis is normalized by Josephson energy $\mathrm{E}_{\mathrm{J}} \mathrm{k}_{\mathrm{B}}$ is the Boltzmann constant. 


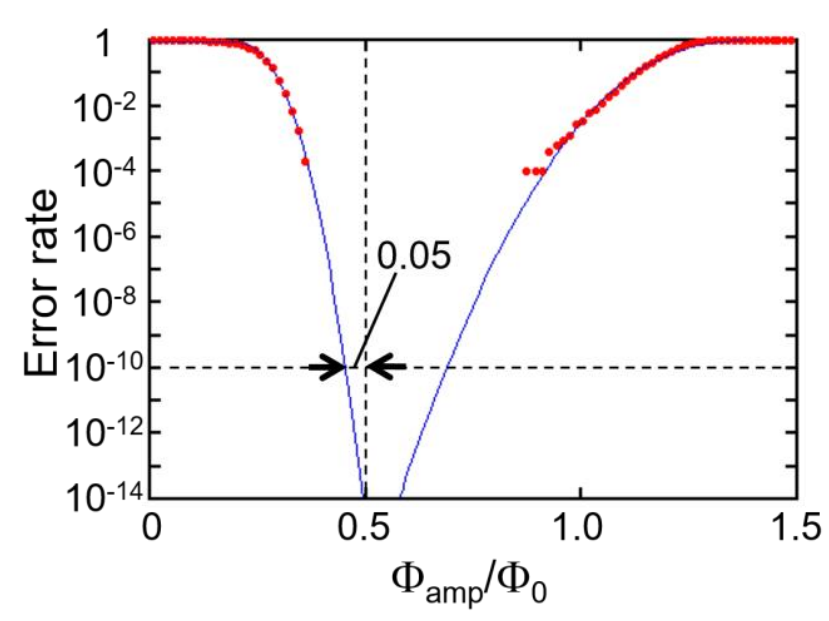

Fig. 4. Simulated dependences of the error rate of the read/write operation of the rf-SQUID memory cell on the input magnetic flux amplitude $\Phi_{\text {amp }}$ when $\mathrm{k}_{\mathrm{B}} \mathrm{T} / \mathrm{E}_{\mathrm{J}}$ is 0.42 . The horizontal axis is normalized by single flux $\Phi_{0} . \mathrm{k}_{\mathrm{B}}$ is the Boltzmann constant. Solid curves are fitting characteristics based on the error function.

operation without applying noise is $0.5 \Phi_{0}$ and the power dissipation is proportional to the square of the input magnetic flux amplitude $\Phi_{\text {amp }}\left(\left(0.45 \Phi_{0} / 0.5 \Phi_{0}\right)^{2}=0.81\right)$. Though the power consumption from noise is not taken into account in this estimation, noise input from room-temperature instruments is not necessary by using internal noises such as thermal noises.

Fig. 4 shows simulated dependences of the error rate of write/read operation on the input magnetic flux amplitude $\Phi_{\text {amp }}$ when $k_{B} T / E_{J}$ was the optimum value of 0.42 . The error rate characteristic $(E R)$ can be fitted by using the error function represented by

$$
E R\left(\Phi_{\text {amp }}\right)=(1 / 2)\left[1+\operatorname{erf}\left\{\left(\Phi_{\text {amp }}-\Phi_{m}\right) / \Phi_{s}\right\}\right],
$$

where $\Phi_{\mathrm{m}}$ is the magnetic flux value of $\Phi_{\text {amp }}$ when the error rate is $0.5, \Phi_{\mathrm{s}}$ is the equivalent flux noise induced by thermal fluctuation, and $\mathrm{k}_{\mathrm{B}}$ is the Boltzmann constant. When $\Phi_{\text {amp }}$ of 0.45 is used, the error rate of approximately $10^{-10}$ is obtained. Use of stochastic resonance for superconducting circuit operation could contribute to not only reduction in power of the superconducting RAM systems but also stabilization of the low-power SFQ circuits with low critical current Josephson junctions [20].

\section{EXPERIMENTAL}

We designed the 1-bit rf-SQUID memory cell using the AIST $2.5 \mathrm{kA} / \mathrm{cm}^{2} \mathrm{Nb}$ standard process 2 [21], and tested the SQUID memory cell by applying noise from a room-temperature instrument. The InductEX [22] was used for extraction of self- and mutual inductance of the designed circuit. Fig. 5 shows an equivalent circuit schematic of the implemented rf-SQUID memory cell and the test setup. A properly biased dc-SQUID is used to detect the state transition of the memory cell. The differential amplifier amplifies the output voltage from the dc-SQUID. The external magnetic flux

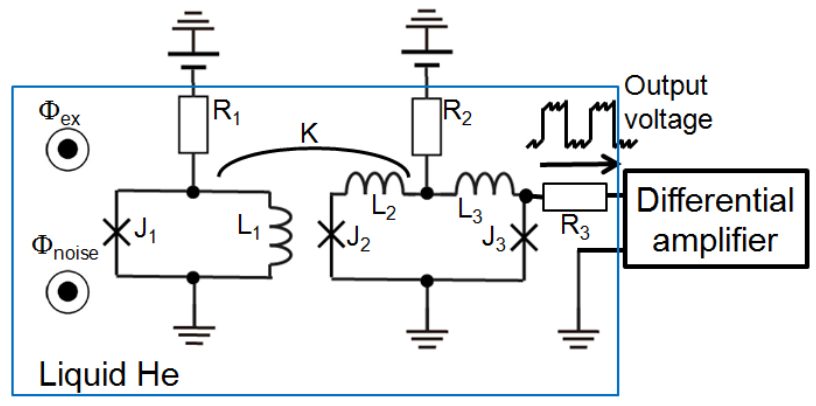

Fig. 5. Circuit schematic of the measured rf-SQUID memory cell. The dc-SQUID detects datum write/read. The external magnetic flux $\Phi_{\text {ex }}$ and the noise magnetic flux $\Phi_{\text {noise }}$ are applied to the rf-SQUID from a room-temperature instrument. $J_{I}=50 \mu \mathrm{A}, J_{2}=J_{3}=100 \mu \mathrm{A}, L_{1}=30.3 \mathrm{pH}, L_{2}$ $=L_{3}=3.5 \mathrm{pH}, R_{l}=R_{2}=500 \Omega, R_{3}=7.5 \Omega, \mathrm{k}=0.3$ is magnetic coupling coefficient between $L_{1}$ and $L_{2}, L_{3}$.

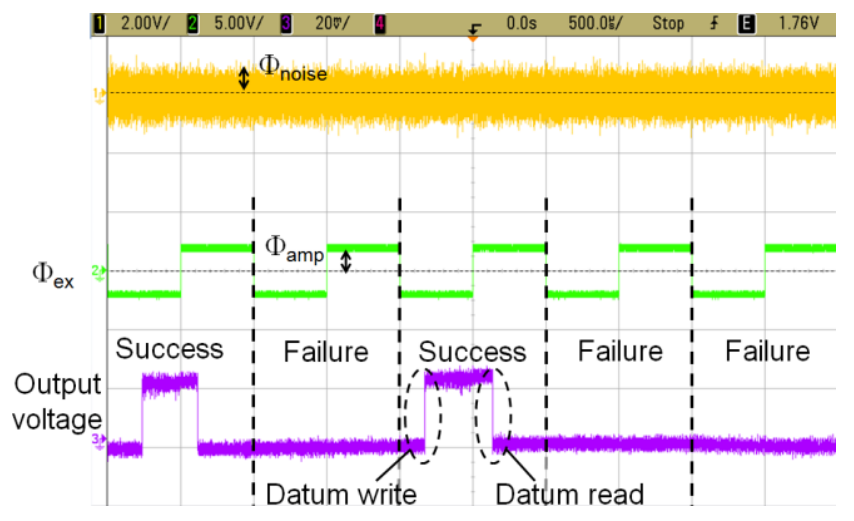

Fig. 6. Measured waveform of write/read operation of the rf-SQUID memory cell. The dc-SQUID is properly biased to detect datum write/read. $\Phi_{\mathrm{ex}}$ is the inverse of the current supplied to the memory cell. $\Phi_{\mathrm{amp}} / \Phi_{0}$ was 0.25 in this measurement. Because the noise condition was not optimum, long delay of datum wite was observed.

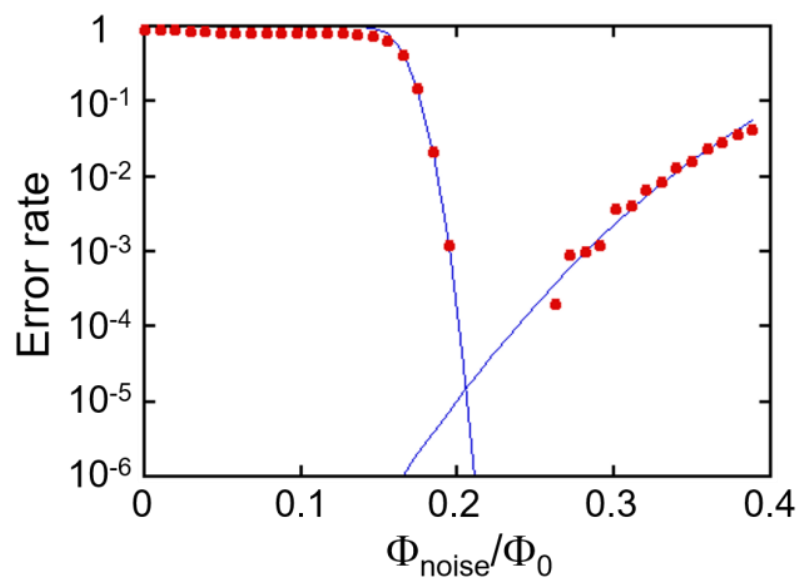

Fig. 7. Dependence of the measured error rate of the read/write operation of the 1-bit rf-SQUID memory cell on the amplitude of the standard deviation of magnetic flux noise $\Phi_{\text {noise. }}$ Dots are measurement results. Solid curves are fitting characteristics.

$\Phi_{\text {ex }}$ and the magnetic flux noise $\Phi_{\text {noise }}$ were applied by arbitrary wave generators. The applied noise was white noise with the bandwidth of $20 \mathrm{MHz}$. Fig. 6 shows the measured waveform. We repeated $10000 \mathrm{read} / \mathrm{write}$ operations at the frequency of 1 $\mathrm{kHz}$. By monitoring the output voltage from the dc-SQUID, the 
error rate was experimentally evaluated using an automated measurement system based on a LabVIEW program.

Fig. 7 shows the dependence of the measured error rate on the standard deviation of the applied magnetic flux noise $\Phi_{\text {noise }}$ when $\Phi_{\text {amp }}=0.45 \Phi_{0}$. We obtained the characteristic similar to the simulated one. The experimentally obtained lowest error rate was approximately $10^{-5}$. In this case, power consumption of write/read operation was reduced by approximately $20 \%$. Discrepancy of the lowest error rate between simulated and measured results is thought to be caused by difference of the operations frequency and the noise bandwidth between simulation and experiment. The ratio between the noise bandwidth and input frequency was 20000 in the measurement, whereas the ratio is 10000 in circuit simulation. High noise power might deteriorate the measured error rate.

\section{CONCLUSION}

We investigated reduction in power consumption of $\mathrm{read} /$ write operation of the rf-SQUID memory cell using stochastic resonance. Simulation results show that stable $\mathrm{read} / \mathrm{write}$ operation can be expected with reduced power consumption by using noise-assisted state transition. We designed and tested the rf-SQUID memory cell, and experimentally observed clear stochastic resonance. By introducing stochastic resonance, total power consumption of the superconducting RAM can be reduced by approximately $20 \%$ compared to that of the conventional superconducting Josephson RAM.

\section{ACKNOWLEDGMENT}

The devices were fabricated in the clean room for analog-digital superconductivity (CRAVITY) in National Institute of Advanced Industrial Science and Technology (AIST).

\section{REFERENCES}

[1] TOP500. [Online]. Available: http://www.top500.org/

[2] D. S. Holmes, A. L. Ripple, and M. A. Manheimer, "Energy-Efficient Superconducting Computing - Power Budgets and Requirements," IEEE Trans. Appl. Supercond., vol. 23, no. 3, 2013, Art. ID. 1701610.

[3] M. M. Manheimer, "Cryogenic Computing Complexity Program: Phase 1 Introduction," IEEE Trans. Appl. Supercond., vol. 25, no. 3, June 2015. Art. ID. 1301704.

[4] Q. P. Herr, A. Y. Herr, O. T. Oberg, and A. G. Ioannidis, "Ultra-low-power superconductor logic," J. Appl. Phys., vol. 109, May 2011, Art. ID. 103903.
[5] M. Tanaka, M. Ito, A. Kitayama, T. Kouketsu, and A. Fujimaki, "18-GHz, 4.0-aJ/bit Operation of Ultra-Low-Energy rapid Single-Flux-Quantum Shift Registers," Jpn. J. Appl. Phys., vol. 51, May 2012. Art. ID. 053102.

[6] D. E. Kirichenko, S. Sarwana, and A. F. Kirichenko, "Zero Static Power Dissipation Biasing of RSFQ Circuits," IEEE Trans. Appl. Supercond., vol. 21, no. 3, pp. 776-779, June 2011.

[7] M. H. Volkmann, A. Sahu, C. J. Fourie, and O. A. Mukhanov, "Implementation of energy efficient single flux quantum digital circuits with sub-aJ/bit operation," Supercond. Sci. Technol., vol. 26, Nov. 2012, Art. ID. 015002.

[8] N. Takeuchi, Y. Yamanashi, and N. Yoshikawa, "Measurement of $10 \mathrm{zJ}$ energy dissipation of adiabatic quantum-flux-parametron logic using a superconducting resonator," Appl. Phys. Lett., vol. 102, Jan. 2013, Art. ID. 052602 .

[9] P. F. Yuh, "A 2-kbit superconducting memory chip," IEEE Trans. Appl. Supercond., vol. 3, no. 2, pp. 3013-3021, June 1993.

[10] P. F. Yuh and P. Bradley, "One-junction superconducting memory cell with column sense," IEEE Trans. Appl. Supercond., vol. 5, no. 3, pp. 3459-3463, Sep. 1995.

[11] S. Nagasawa, Y. Hashimoto, H. Numata, and S. Tahara, "A 380 ps, 9.5 $\mathrm{mW}$ Josephson 4-kbit RAM operated at a high bit yield," IEEE Trans. Appl. Supercond., vol. 5, no. 2, pp. 2447-2452, 1995.

[12] T. Ortlepp and T. Van Duzer, "Access time and power dissipation of a model 256-bit single flux quantum RAM," IEEE Trans. Appl. Supercond.,vol. 24, no. 4, 2014, Art. ID. 1300307.

[13] I. V. Vernik, V. V. Bol'ginov, S. V. Bakurskiy, A. A. Golubov, M. Y. Kupriyanov, V. V. Ryazanov, and O. A. Mukhanov, "Magnetic Josephson Junctions With Superconducting Interlayer for Cryogenic Memory," IEEE Trans. Appl. Supercond., vol. 23, no. 3, June 2013, Art. ID. 1701208 .

[14] B. M. Niedzielski, S. G. Diesch, E. C. Gingrich, Y. Wang, R. Loloee, W. P. Pratt, and N. O. Birge, "Use of Pd-Fe and Ni-Fe-Nb as Soft Magnetic Layers in Ferromagnetic Josephson Junctions for Nonvolatile Cryogenic Memory," IEEE Trans. Appl. Supercond., vol. 24, no. 4, Mar. 2014, Art. ID. 1800307.

[15] B. Baek, W. H. Rippard, S. P. Benz, S. E. Russek, and P. D. Dresselhaus,, "Hybrid superconducting-magnetic memory device using competing order parameters," Nat. Commun., vol. 5, May 2014, Art. ID. 3888.

[16] L. Ye, D. B. Gopman, L. Rehm, D. Backes, G. Wolf, T. Ohki, A. F. Kirichenko, I. V. Vernik, O. A. Mukhanov, and A. D. Kent, "Spin-transfer switching of orthogonal spin-valve devices at cryogenic temperatures," J. Appl. Phys., vol. 115, Feb. 2014, Art. ID. 17C725.

[17] L. Gammaitoni, P. Haenggi, P. Jung, and F. Marchesoni, "Stochastic resonance," Rev. Mod. Phys., vol. 70, no. 1, pp. 223-288, Jan. 1998.

[18] A. D. Hibbs, A. L. Singsaas, E. W. Jacobs, A. R. Bulsara, J. J. Bekkedahl, and F. Moss, "Stochastic resonance in a superconducting loop with a Josephson junction," J. Appl. Phys. Vol. 77, pp. 2582-2590, 1994.

[19] J. Satchell, "Stochastic simulation of SFQ logic," IEEE Trans. Appl. Supercond., vol. 7, no. 2, pp. 3315-3318, Jun. 1997.

[20] M. Tanaka, A. Kitayama, T. Koketsu, M. Ito, and A. Fujimaki, "Low-Energy Consumption RSFQ Circuits Driven by Low Voltages," IEEE Trans. Appl. Supercond., vol. 23, no. 3, June 2013, Art. ID. 1701104.

[21] M. Hidaka, S. Nagasawa, T. Satoh, K. Hinode and Y. Kitagawa, "Current status and future prospect of the Nb-based fabrication process for single flux quantum circuits," Supercond. Sci. Technol., vol.19, pp. S138-S142, 2006.

[22] C. J. Fourie, O. Wetzstein, T. Ortlepp, and J. Kunert, "Three-dimensional multi-terminal superconductive integrated circuit inductance extraction," Supercond. Sci. Techonol., vol. 24, Nov. 2011, Art. ID. 125015. 